\title{
¿De qué infancias hablan los educadores del nivel inicial?'1
}

Rosa Julia Guzmán Rodríguez²

\section{Introducción}

En algunos estudios recientes y en otros no tan recientes se habla de que los educadores de primera infancia llegan a las aulas de clase y a las salas del nivel inicial con las concepciones de infancia que se han configurado en los ámbitos sociales en que ellos se desenvuelven, y que éstas les sirven de soporte para desarrollar determinadas acciones con los niños y las niñas. Así mismo, se considera que las actividades reflejan dichas concepciones de infancia; esto es, la escuela o el jardín infantil se constituyen en instituciones receptoras y reproductoras de lo simbólico de la cultura.

En este artículo queremos mostrar otra perspectiva, con base en los resultados de un estudio realizado por el Grupo de Investigación en Infancia ${ }^{3}$ de la Facultad de Educación de la Universidad de La Sabana, con educadoras del nivel inicial en Chía.

Partimos de reconocer que, como se señaló anteriormente, la construcción del concepto de infancia se ubica en el universo simbólico de las culturas y, por tanto, tiene una fuerte incidencia en las acciones que se emprenden en torno a las niñas

\footnotetext{
1 Texto recibido el 9 de agosto de 2007, evaluado el 10 y 13 de septiembre y arbitrado el 30 de octubre 2007.

2 Doctora en Educación, Universidad de Nova de Lisboa. Investigadora y profesora, Facultad de Educación Universidad de La Sabana. Directora Línea de Investigación en infancia, que hace parte del Grupo de investigación Educación y educadores, reconocido por Colciencias. rosa.guzman@unisabana.edu.co

3 El Grupo está conformado además por las profesoras Sandra Téllez, Sandra Sánchez, Esther Susana Martínez, Mónica Guevara y Rosario Bernal. Son miembros del semillero de investigación, Alejandra Cifuentes, estudiante de Maestría y las estudiantes de Pedagogía Infantil Gloria Bernal, Érika Alarcón, Viviana Botero, Victoria Torres y Ana María Cuartas.
} 
y los niños. La educación y la pedagogía se convierten, por supuesto, en espacios sociales fuertemente afectados por estas concepciones.

Igualmente, asumimos que a lo largo de la historia las propuestas educativas y pedagógicas han variado de acuerdo con la evolución que ha tenido el concepto de infancia y con los contextos en que se trabajan y se llevan a cabo prácticas específicas de atención y educación a los niños y las niñas, que se hacen visibles en diferentes espacios, como la sociedad en general, la familia, los hogares comunitarios, los jardines y la escuela.

Sin embargo, consideramos que la escuela y otros espacios educativos institucionales, tales como los hogares comunitarios y los jardines infantiles, entendidos no solamente como el espacio físico, sino también simbólico, configuran nuevas concepciones de infancia. Esto sucede porque la concepción en cuanto construcción de la realidad es una guía para la acción y, a la vez, determina los comportamientos o prácticas de los individuos. Pero esta relación no es lineal porque la concepción se ha construido también mediante prácticas instituidas, que se vuelven constitutivas e identitarias de grupos y comunidades.

De esta manera, en las instituciones de formación inicial, puede haber prácticas y comportamientos hacia la infancia que son parte de su manera espontánea y natural de concebir la relación con los niños, concepción que pertenece a una tradición y que se asienta como práctica cultural. En términos generacionales y de transmisión de un sistema de normas y valores, estas prácticas, a su turno, posibilitan cosmovisiones que promueven nuevamente determinadas acciones relacionadas con dicha concepción.

En este orden de ideas, si bien las concepciones orientan la acción, comportamiento y prácticas de los individuos y grupos, también puede decirse que expresan prácticas sedimentadas que dan cabida a las interpretaciones del mundo por parte de los actores educativos. Desde este punto de vista, las concepciones y las prácticas se generan mutuamente, se arraigan en un pasado colectivo y se actualizan en la vida cotidiana. Dada la fuerza de las concepciones en la vida social, es importante investigar cuáles son las concepciones de infancia de las educadoras del nivel inicial y ampliar este conocimiento desde la óptica teórica y de aplicación práctica.

Desde esta perspectiva, la Facultad de Educación de la Universidad de La Sabana planteó la investigación Concepciones de infancia que tienen los Educadores del nivel inicial en Chía. Su propósito central es indagar sobre las concepciones de infancia que tienen las madres comunitarias, las educadoras de los jardines infantiles y las profesoras de transición en este municipio. Es un estudio de tipo exploratorio, que se asume como la primera etapa de un proceso de investigación más amplio.

La pregunta de investigación planteada fue ¿Qué concepciones de infancia tienen las madres comunitarias, las educadoras de jardín infantil y de transición, en el municipio de Chía?

Se trabajó en Chía, por pertenecer al área de influencia de la Universidad de La Sabana. Éste es un municipio cercano a la capital del país, que a pesar de mantener 
en algunos sectores una vocación agrícola y ganadera, se convirtió en un sitio apetecido para vivir cerca de Bogotá (la capital del país), pero fuera de ella, lo que ha incrementado su urbanismo.

Para identificar las concepciones de infancia de educadores del municipio de Chía, se hizo una investigación cualitativa, ya que su pretensión es revelar el sentido que los educadores les dan a sus prácticas cotidianas y las concepciones explícitas de infancia, que desde su perspectiva, las sustentan. De esta manera entendemos que las prácticas son acciones, intencionales o no, que se producen en contextos cotidianos de interacción, en los cuales adquieren significado.

Los educadores con quienes se efectuó la investigación para construir el objeto de conocimiento (concepciones de infancia que tienen los educadores del nivel inicial en Chía) fueron seleccionados intencionalmente, considerando que los diferentes estratos y las distintas naturalezas de las instituciones estuvieran cubiertos, así: instituciones privadas de estratos, alto y medio, e instituciones oficiales. Dentro de cada institución se seleccionó la población de educadores, teniendo en cuenta aquellos que no tienen formación académica (madres comunitarias) y aquellos con formación (docentes). Así, se asegura que estén representadas características dentro de la población que, desde nuestra perspectiva, pueden ser reveladoras con respecto a las concepciones de infancia que configuran los educadores del nivel inicial.

El tiempo de experiencia de las educadoras oscilaba entre los dos y más de veinte años y sus edades fluctuaban entre los 24 y los 45 años, todas ellas mujeres; algunas provenían del sector rural y otras del sector urbano de Chía y de Bogotá.

Las educadoras respondieron un cuestionario, a partir de cuyas respuestas se crearon varias categorías; éstas permitieron ampliar las preguntas planteadas en un segundo momento de indagación, por medio de entrevistas semiestructuradas individuales. Con todas las categorías que surgieron en estos primeros niveles de análisis, se estructuró una guía para trabajar con un grupo focal.

La información obtenida mediante los instrumentos antes mencionados se organizó en matrices de análisis para hacer nuevas lecturas de ésta, con el fin de comprender mejor el objeto de estudio.

Por concepciones de infancia se entienden las ideas, razones, justificaciones, creencias, opiniones, mitos, metáforas, con ayuda de los cuales se alude al niño o niña menor de siete años. Estas concepciones se desarrollan en el seno de los procesos sociales que viven los individuos y pueden proceder tanto del sentido común, como de un saber más organizado.

Por prácticas educativas se entienden las acciones que se proponen en el marco de la educación, cargadas de una intención formadora hacia la población infantil. Esas acciones ocurren en procesos de interacción y en el aula de clase, entendida, en un sentido amplio, como todo ese espacio físico y simbólico que configura el trabajo en las instituciones encargadas de esta función educadora. 


\section{Referente teórico}

\section{Concepciones sociales}

Partimos de asumir el término concepciones, tal como lo plantean Zimmerman y Gerstenhaber (2000):

Por concepción se entiende un proceso personal por el cual un individuo estructura su saber a medida que integra sus conocimientos. Este saber se elabora, en la mayoría de los casos, durante un periodo bastante amplio de la vida, a partir de su arqueología; es decir, de la acción cultural parental, de la práctica social del niño en la escuela, de la influencia de los diversos medios de comunicación y, más tarde, de la actividad profesional y social del adulto. Las concepciones personales son la "única trama de lectura” a las que se puede apelar cuando se confronta con la realidad (p. 125).

En este orden de ideas, se asume en la presente investigación que desde el conocimiento de las concepciones de los educadores de primera infancia se pueden comprender las prácticas educativas cotidianas, al igual que las razones, motivos, creencias y supuestos que las orientan, ya que como plantean Giordan y De Vecchi (citados por Zimmerman y Gerstenhaber, 2000):

El término concepción personal enfatiza la idea de imágenes coordinadas entre sí que son usadas por las personas para razonar frente a situaciones problema. Toda concepción, afirman, se corresponde con una estructura subyacente y no es sólo un producto sino un proceso que depende de un sistema que constituye su marco de significación con el que las personas intentan interpretar su medio. Las concepciones tienen una génesis que es al mismo tiempo individual y social (p. 125).

El principal aporte de esta investigación consiste en poner en el centro de las discusiones y análisis educativos las concepciones de infancia que tienen las educadoras del nivel inicial, como fuente importantísima para los procesos de atención y educación a la primera infancia. En esto coincidimos con Zimmerman y Gerstenhaber (2000), cuando afirman:

En este sentido, conocer las concepciones personales de los docentes puede permitirnos replantear muchas de nuestras acciones por mejorar los procesos de enseñanza y aprendizaje en la sala del nivel inicial. Esta afirmación no responde a una intención técnica que intenta reemplazar concepciones "defectuosas" por otras "correctas", sino a una perspectiva que propone abordar la práctica educativa como un fenómeno complejo, en un contexto particular y en el cual participan muchos factores (p. 126).

El abordaje del objeto de estudio (concepciones de infancia que tienen las educadoras del nivel inicial en Chía) se plantea en esta investigación desde un marco pedagógico, que le da una perspectiva de análisis diferente de la que podría hacerse desde la sociología, la psicología o cualquier otra disciplina. Podría afirmarse que 
las concepciones que tienen los agentes educativos sobre infancia son consecuentes con la actitud o comportamiento hacia la práctica educativa. Hacer depender las actitudes y comportamientos hacia la infancia, de las concepciones sobre ella, significa en otras palabras, que los actores educativos poseen una visión del mundo que les permite dar sentido a sus conductas relacionadas con niño y niña, así como entender la realidad infantil a través de un determinado sistema de referencias. Las concepciones construidas por dichos actores están integradas a su sistema de valores, construcción que se ha realizado a lo largo de la vida y en interacción con el contexto sociocultural en que se desarrolla.

\section{Concepciones de infancia}

Las concepciones de infancia han sido bastante variables. Han cambiado según la época histórica que se viva y, así mismo, según el sector desde donde se analicen. A este respecto, Mami Umayahara (2003), especialista asistente del programa Unesco, Oficina Regional de Educación para América Latina y el Caribe, afirma:

Existe un consenso sobre la definición de infancia que se refiere a la población menor de 18 años, reflejando la adopción de la Convención Internacional de los Derechos de los niños y las niñas. Sin embargo, cuando se refiere a la primera infancia, existe una variedad en cuanto a los tramos de edad. Hay un acuerdo en que la primera infancia comienza con el nacimiento, pero no se sabe cuándo termina; en general, la mayor incidencia es hasta los seis años de edad y en algunos países contemplan hasta cinco años. En algunos países, el sector salud suele utilizar cinco años porque la $\mathrm{OMS}^{4}$ utiliza cinco años como un umbral importante de sobrevivencia de los niños. Por otro lado, el sector educación suele utilizar seis años, porque ahí comienza normalmente la educación primaria. En el caso de Colombia es un poco excepcional, porque el $\mathrm{ICBF}^{5}$ cubre desde cero a siete años, mientras que el sector educativo se preocupa más a partir de cinco años (p. 170).

Sobre este particular, Carlina Rinaldi (citada por Peralta, 2003) señala lo siguiente: "La infancia no existe. La creamos como sociedad, como un tema público. Es una construcción social, política e histórica” (p. 118). Por otra parte, Peter Moss (citado por Peralta, 2003) sostiene: "La idea de un niño universal, conocible objetivamente y separado de su tiempo y espacio, contexto y perspectiva, ha estado cuestionándose crecientemente” (p. 118).

Estas afirmaciones llevan a pensar que la formación de educadores de niños y niñas debe, necesariamente, incluir estas reflexiones porque es común encontrar, tanto en los discursos cotidianos como en los académicos de los educadores, muchas referencias a la intención de lograr que sus alumnos lleguen a ser, niños ideales, o

\footnotetext{
${ }^{4}$ Organización Mundial de la Salud.

${ }^{5}$ Instituto Colombiano de Bienestar Familiar.
} 
por lo menos se acerquen mucho a alcanzar estas condiciones, generando incluso discursos con los que se encubren posiciones discriminatorias y excluyentes frente a algunos sectores de la población infantil.

En la actualidad es absolutamente clara la pertinencia del trabajo durante los primeros años de vida para el desarrollo de los individuos y de las sociedades. Esta pertinencia está relacionada con las acciones que se emprendan con los pequeños, puesto que determinan en gran medida el tipo y nivel de desarrollo posterior. En tal sentido, las neurociencias han hecho importantes aportes, al señalar el gran potencial neuronal y de sinapsis con que cuentan los recién nacidos. Goulart (citado por Peralta, 2003) destaca que "La ejercitación y las prioridades que se establecen hacen una estabilización selectiva de estas conexiones, a través de las cuales se organiza el mundo exterior" (p. 125).

Por su parte, Edelman (citado por Peralta, 2003) manifiesta que "son el contexto y la historia del desarrollo celular de un individuo los que determinan en gran parte la estructura de su cerebro y no la mera información genética” (p. 126). Así mismo, Peralta (2003) asegura que las investigaciones en neurociencias "conllevan un enorme desafío tanto para los padres como para los encargados de generar políticas educativas y para los educadores que las implementan” (p. 127). A este respecto, Prout y James (citados por Peralta, 2003) afirman: "La inmadurez de los niños es un eje biológico, pero las formas en que esta inmadurez es entendida y se les da significado es un hecho de la cultura". Agrega Peralta (2003): "Por tanto, la infancia es una construcción social críticamente afectada por las ideas, modas y tecnología con que se cuenta" (p. 136).

Estas afirmaciones son particularmente importantes en el campo de la educación, ya que las niñas y los niños pasan una etapa y un tiempo esenciales de su vida en instituciones de educación inicial y en la escuela, y en su interior existen ideas, tendencias, creencias y tecnologías que inciden en las formas de educación que se les imparte.

Esto se hace evidencia al revisar estudios sobre la historia de la educación y la pedagogía, como los efectuados por Olga Lucía Zuluaga (1987) y Javier Sáenz y Óscar Saldarriaga (1997), en los que se plantea cómo el manejo pedagógico y el trato dados a los estudiantes en la escuela han variado de acuerdo con las ideas que se tenían con respecto a lo que era un niño o una niña. Estas ideas han servido de soporte a la generación de las formas en que se les debe educar y a las estrategias con que se les debe enseñar. Por otro lado, los estudios de Muñoz y Pachón (1991) sobre la infancia del siglo XX, aunque se han efectuado desde otra perspectiva, también han hecho aportes que han permitido conocer la situación de los niños y niñas en general y de los escolarizados en particular.

En varios estudios (Muñoz y Pachón, 1991; Sáenz y Saldarriaga, 1987; Delgado, 2000) se plantea que han existido diversas concepciones de infancia, según la clase social a la que pertenecieron los niños y las ciencias, fueran o no escolarizados, e incluso dentro de la institución escolar se consideraban diferentes según su nivel 
socioeconómico de procedencia. En el sistema educativo de principios del siglo XX se presentaban claras distinciones entre la educación para los niños ricos y la educación para los niños pobres. Citan Muñoz y Pachón (1991) una afirmación del Registro Municipal de Bogotá, del 30 de junio de 1920, en la que se habla de la necesidad de dar en la escuela para pobres "conocimientos especiales que les sean útiles para las posiciones de campesinos y obreros, que son las que ocuparán siempre” (p. 119).

Esta tendencia persiste incluso hasta nuestros días y es lo que sustenta ideas tales como que los niños de estratos bajos están condenados al fracaso. Desde hace ya bastante tiempo se conocen estudios muy serios, en los que se demuestra que las bajas expectativas sobre este sector de la población infantil llevan a que, en efecto estos estudiantes aprendan poco y en consecuencia fracasen en la escuela y en la sociedad (Ferreiro y Teberosky, 1979).

\section{Escuela y concepciones de infancia}

A partir de los aportes hechos por Postman (2004), se sabe que la institución escolar ha influido también de manera clara en la construcción social de diversas concepciones de infancia. Afirma el autor que, desde hace mucho tiempo, los intereses por alfabetizar a los niños generaban nuevas concepciones de infancia: "En los sitios donde se valoraba persistentemente la alfabetización, se construían escuelas, y donde había escuelas, el concepto de niñez evolucionaba rápidamente” (p. 17).

Con el paso del tiempo la institución escolar empezó a ofrecer a las sociedades, de modos diferentes, concepciones de infancia que respondían a sus ideas acerca de cómo debían organizarse las escuelas para educar a los niños. Fue así como en las primeras etapas se clasificaba a los niños por su destreza en la lectura y no por la edad. Como la enseñanza escolar se reducía a dar los conocimientos básicos en lectura, escritura y matemáticas, la infancia se reconoció como la etapa escolar y culminaba alrededor de los doce años. Más tarde, se empezaron a diseñar los currículos como respuesta a las necesidades que planteaban los diferentes niveles de desarrollo de los niños y las niñas, que posteriormente se identificaron con edades muy definidas para cada grado, desconociendo las posibilidades individuales y las necesidades provenientes de los distintos contextos.

Estas ideas en torno a las necesidades de los niños y las niñas se conocieron también en Colombia. Sáenz y Saldarriaga (1997) cuentan que en el Gimnasio Moderno, en 1917, siguiendo las enseñanzas de María Montessori, se abrió una sección para los niños de tres a cinco años, asumiendo que ésta implicaba un cambio profundo del concepto de niño y de sus necesidades. El anterior es un ejemplo claro de la forma en que la escuela, con sus dinámicas propias, aporta diversas concepciones de infancia a la sociedad. Las diferencias en Colombia no se percibían solamente por razones de edad; también había diferencias explícitas en la educación por razones de género.

En un documento del Ministerio de Educación Nacional sobre la educación preescolar (1996) se señalan los momentos por los que ha pasado este nivel edu- 
cativo en Colombia: primero fue un modelo asistencial, luego se les dio un énfasis educativo sin reconocimiento legal en el ámbito gubernamental, después tuvo vida legal y contó con un diseño curricular, y por último se impuso el grado de transición como obligatorio.

A pesar de estos pasos para reconocer la necesidad y la importancia de dar educación a la primera infancia, el camino no ha sido claro. Se han hecho diversos esfuerzos para atender de la mejor manera posible a los niños y las niñas, pero persisten algunas dificultades derivadas de las concepciones que se tienen de lo que es un niño o una niña de esta edad y de lo que está en capacidad de hacer. Actualmente hay varios interrogantes referentes a lo que se debe hacer en el preescolar y lo que se debe hacer en el primer grado. Allí sigue existiendo una ruptura entre las propuestas de trabajo, como si entre estos dos grados existieran años de diferencia. En el fondo, son concepciones diferentes y discontinuas de niño o niña preescolar y niño o niña escolar.

En estas búsquedas se ha recurrido a las comprensiones que tienen otras disciplinas de lo que es ser niña o niño y se ha intentado aplicarlas a la educación. Sin embargo, dichas aplicaciones no siempre han dado buenos resultados, lo que evidencia la importancia de tomar en cuenta las comprensiones que tienen los educadores de lo que es ser niño o niña, para introducir cambios en la educación.

\section{Las concepciones de infancia que tienen las educadoras del nivel inicial en Chía ${ }^{6}$}

Las concepciones de infancia, explícitas e implícitas, que tienen las educadoras del nivel inicial en Chía se indagaron a partir de varios aspectos, con el propósito de articular las interpretaciones dadas a cada uno de ellos. Por tal razón se investigó sobre las ideas que tienen las educadoras sobre lo que implica educar a los niños y las niñas, ya que éstas permiten hacer aproximaciones a concepciones que han configurado acerca de la infancia. Si bien algunas de las categorías creadas a partir del análisis de información aluden a las educadoras y a sus motivaciones individuales, también aportan información sobre sus concepciones de infancia, en cuanto se refieren a lo que desde su perspectiva se requiere para educar a la infancia. Por otra parte, cobran importancia en la medida en que en este estudio se busca comprender de qué maneras la escuela contribuye a configurar concepciones de infancia.

\section{Algunos hallazgos}

\section{La infancia como sujeto de prácticas}

Todas las referencias que se hacen a la motivación para ser educadoras tienen que ver con experiencias personales o familiares, que remiten claramente a situaciones

\footnotetext{
${ }^{6}$ Se habla de educadoras porque todas son mujeres
} 
prácticas. En ninguna circunstancia se habló de algún aspecto teórico, lo que llama especialmente la atención en el caso de las educadoras con formación académica y plantea a la vez la necesidad de indagar si esto obedece a que existe una percepción de la educación infantil como práctica que se aprende fundamentalmente mediante la acción directa con los niños y las niñas, o si remite a un encuentro afectivo directo entre los adultos y ellos. También pueden ser las dos razones; es decir, que la infancia no sería sujeto de estudio.

\section{La infancia como sujeto de cuidados, afecto y formación en valores, para el futuro}

Las educadoras consideran que lo más importante en su trabajo es la formación de valores en los niños y las niñas, entendido esto como educarlos para mantener los valores existentes actualmente en la sociedad. Es curioso que en tal reconocimiento de los valores se presuma que éstos son los deseables y garantes del buen funcionamiento de las sociedades, mientras que se critica la situación actual. Tal vez se refieran a una situación ideal, basada en la añoranza de épocas anteriores.

Se destaca sobremanera la alusión a asumir el papel de segunda madre con los niños y las niñas, y de dar afecto permanente. Las educadoras piensan que ésta es una de sus principales funciones; incluso pueden obviar las demás. Para ellas es clara la idea de que los niños y las niñas necesitan el afecto de los adultos que los cuidan y educan, para poder dar continuidad al hogar y facilitar la salida de éste.

Para estas educadoras resulta obvio que se educa para el futuro; incluso cuando hablan de la importancia de la primera infancia en el desarrollo de los individuos, se remiten a que "pueden forjar buenas bases para que los niños tengan un mejor futuro", con lo que se refieren en su gran mayoría a la vida laboral.

Con respecto a estas ideas se encuentra una contradicción cuando expresan que quieren dar continuidad al hogar, pero manifiestan tener dificultades con los padres de familia porque "quieren imponer sus puntos de vista", "son conflictivos", "son despreocupados", "no valoran nuestro trabajo". Incluso mencionan que algunas veces las propuestas pedagógicas que plantean se ven interferidas por las actitudes de los niños, ocasionadas por "la triste vida que llevan". Cabrían entonces las siguientes preguntas: ¿por qué querer dar continuidad a los hogares de los niños y las niñas en la institución? ¿Estarán pensando en una familia ideal o quieren proporcionar en la institución lo que el hogar, según ellas, no da? Lo que sí es claro es la concepción de infancia como sujeto de protección y cuidados.

\section{La infancia como sujeto de prácticas fáciles de educación}

En las referencias directas a los niños y las niñas con quienes trabajan, señalan como motivos de su preferencia por trabajar con ellos y no con más grandes, que es más fácil controlarlos y enseñarles, porque saben menos y están dispuestos a aprender 
cualquier cosa; se puede reemplazar a la mamá y es posible brindarles cariño. Esta idea de que es más fácil trabajar con los más pequeños es bastante relativa, si se considera el fuerte impacto que tiene la educación en edades tempranas y la gran variedad de actividades que se requiere preparar tanto para tomar en cuenta los periodos breves de atención que se les prestan a los pequeños, como para ocuparse de la influencia que tienen las actividades propuestas en el nivel de desarrollo que se alcance en los niños y las niñas. Quizá se asuma, en este caso, la educación como el cuidado físico, la atención afectiva y el mantenerlos entretenidos o, por lo menos, ocupados.

\section{La infancia como sujeto de prácticas educativas basadas en la vocación, el amor, la tolerancia, el cariño, el carisma y la entrega, junto con el uso de estrategias lúdicas}

Entre las condiciones que se requieren para tener éxito en la educación de los pequeños, sobresalen la vocación, el amor, la tolerancia, el carisma, la entrega y el cariño. Aunque todas son necesarias, no son suficientes, y no deja de resultar extraño que ninguna mencione los conocimientos adquiridos durante la formación académica como requisito clave. Parecería que éste fuera un saber empírico y una cuestión de buena voluntad. Tal vez ésta sea una de las razones por las que consideran más fácil el trabajo con la primera infancia.

Como una idea complementaria a la anterior, exponen la importancia de trabajar con estrategias lúdicas, pero no mencionan qué se debe trabajar. Es como si significara más la estrategia que el contenido, lo que en el fondo guarda relación con la idea de que los niños y las niñas deben estar entretenidos y contentos; por eso todo ha de ser lúdico. Sin embargo, vale la pena resaltar que la estrategia sirve en la medida en que se tenga claridad acerca de cómo y para qué utilizarla. Que algo sea lúdico no entraña necesariamente que sirva para promover desarrollos.

Este aspecto plantea serias inquietudes a los organismos encargados de la formación y acompañamiento de los educadores del nivel inicial, en cuanto parece haber en ellos cierto conocimiento de los medios (aunque no necesariamente exista claridad sobre cómo obtener provecho de ellos), pero un desconocimiento con respecto a los fines. Las implicaciones de esta situación en la educación en la primera infancia, son importantes: la evaluación de estos procesos podría quedarse en la mirada a lo inmediato (que los pequeños estén contentos y pasen bien), descuidando la calidad de las actividades propuestas, por desconocimiento en la teoría o en la práctica, de las posibilidades de desarrollo que ofrece una intervención adecuada durante la primera infancia. Contrasta esto con la concepción de infancia como preparación para el futuro, en razón de que no hay claridad acerca de cómo propiciar su desarrollo en la escuela. 


\section{La infancia puede ser eterna o puede terminar dentro de la escuela}

La afirmación de que para ellas no es tan claro en qué momento los niños pasan a otra etapa de la vida surge de aseveraciones como: "Los niños nunca dejan de ser niños", "Siempre somos niños porque todos llevamos un niño dentro", "Nunca deberíamos dejar de ser niños". Así mismo, aseguran: "Los cambios no se notan y tampoco se ve que quieran cambiar". Esta primera posición sugiere una concepción de infancia como un estado de las personas, asociado a condiciones ideales que un ser humano nunca debería perder.

Por otra parte, la afirmación "Cambian cuando pasan a primaria y tienen otros juegos y cambian de amigos", lleva a pensar en que un criterio importante para considerar que un niño o una niña empiezan una nueva etapa en su vida, es el paso de un nivel educativo al siguiente.

\section{Las exigencias de acuerdo con el nivel escolar influyen en la configuración de distintas concepciones de infancia entre el preescolar y primer grado}

Esta idea se refuerza con las afirmaciones hechas por las educadoras, que muestran de manera tajante la ruptura que debe haber, según ellas, entre el trabajo en transición y el de primero de primaria: "El niño de primero ya tiene un pensamiento concreto, mientras que al de preescolar hay que explicarle todo una y otra vez"; "En preescolar son las bases del aprestamiento, en tanto que en primero hay que avanzar"; "En primero hay más responsabilidad en tareas, respeto y aseo"; "En preescolar es más juego"; "En preescolar se les está enseñando todo, recalcándole normas; en primero hay más exigencias".

Se sostiene también que en primaria debe haber exigencias diferentes de las existentes en preescolar, en enunciados como los siguientes: "En primaria se debe mantener la atención por periodos más largos"; "Debe tener un comportamiento más adecuado dentro y fuera del establecimiento"; "Es más conocimiento y el juego queda por fuera"; "Se generan más hábitos"; "En primaria hay que ser más estrictos porque lo del preescolar es como una escalerita que va subiendo, entonces todo cambia"; "En preescolar se tolera más y se trabaja el manejo del cuerpo, y que tenga disposición para aprender”.

\section{Las educadoras de infancia en preescolar y en primaria deben tener características diferentes. Algunas tensiones en esta perspectiva}

Señalan estas educadoras que la profesora de preescolar "Debe ser lúdica y dinámica"; "Que les dé mucho cariño y amor a los niños"; "Que se fije si los niños van al baño, si toman la merienda"; "Que tenga en cuenta cada niño". 
En cambio, dicen que a la profesora de primero "No le insistiría tanto en la paciencia, porque el pensamiento de los niños es diferente y ya tienen hábitos creados, no hay que repetir tanto lo mismo"; "Que sea creativa para que los niños no se aburran"; "Que forme en valores e interiorice conceptos específicos para tener muy buenas bases"; "Que tenga mucha información diferente para que los niños puedan consultar"; "Que utilice las lúdicas para que de vez en cuando cambie la clase magistral".

No obstante, algunas educadoras mencionan ideas diferentes. "Transición y 'primerito' son casi lo mismo porque en los dos grados los niños tienen acceso a otras fuentes de información, como la televisión, la Internet y el medio en general"; otra señala que "preescolar es igual a primero y se exige de acuerdo con las capacidades de cada uno", y otra más afirma que "Se enseña lo mismo de acuerdo con las capacidades, pero hay diferencia en los contenidos".

Esta diferencia de posiciones pone de relieve la importancia de tomar en cuenta "las concepciones de infancia que tienen los educadores", en plural, igual que se habla de "las infancias".

\section{No hay una concepción unificada de infancia entre las educadoras del nivel inicial. Hablan de diferentes infancias}

Podemos afirmar, entonces, que las concepciones de infancia que tienen estas educadoras remiten a un niño desvalido, que requiere protección y cuidados, que requiere de manera prioritaria las manifestaciones permanentes de afecto y cariño, la presencia de la mamá, bien sea de la real o de la que encarna la maestra, que necesita juegos y movimiento libre, que requiere que lo formen en los valores que tenía la sociedad en épocas anteriores, que necesita a un adulto que le organice la vida mediante la definición y el cumplimiento de normas y la práctica de hábitos y rutinas, que es un ser en formación que demanda paciencia por parte de los adultos y al que hay que educar con gran esmero para que tenga posibilidades de éxito en el futuro. Curiosamente, ninguna educadora hizo referencia a los géneros; siempre hablaron de "los niños" o "los alumnos". En síntesis, es una infancia desvalida, que necesita afecto, protección y cuidados, y una guía permanente para que tenga un buen futuro.

Llama la atención que a pesar de referirse con frecuencia a que hay que querer al niño y la niña, y tratarlos con afecto y mucho cuidado, no se hace ninguna referencia explícita a la importancia de la calidad de vida presente de los pequeños, como correspondería a una concepción de niñas y niños como sujetos de derechos. Parecería que las condiciones de buen trato se convirtieran en una posibilidad de inversión para el futuro, que es el de la vida plena, y la actual (primera infancia) se constituyera exclusivamente en "la preparación para". 


\section{Relaciones entre las prácticas pedagógicas y las configuraciones de concepciones de infancia}

Ya se anotó que la concepción de infancia no es igual para todas las educadoras con quienes se llevó a cabo la investigación. Se encontraron diferencias entre las concepciones de infancia de las madres comunitarias y las de las profesoras, y entre las mismas profesoras, según estuvieran vinculadas al sector público o al privado, según el grupo etario atendido y según trabajaran con niños y niñas de estratos bajos, medios o altos.

\section{¿De qué infancias hablan las educadoras?}

Las educadoras del nivel inicial hablan de diferentes infancias. En la configuración de dichas concepciones, donde parecen tener mayor peso sus prácticas cotidianas que su formación, influyen diversos factores, como ya se mencionó. Pero antes de mostrar las diferencias en las concepciones de infancia existentes entre las educadoras, vale la pena destacar dos aspectos comunes entre ellas:

1. A los niños y a las niñas se les educa para el futuro. Como ya se dijo, esto sugiere la idea de cierto desconocimiento de la importancia de la calidad de vida y el bienestar presente en la vida de los pequeños. Esta idea aparece más acentuada en las educadoras que en las madres comunitarias, puesto que están dispuestas a aceptar e incluso a promover "el trabajo duro" de los estudiantes a cambio de garantizar un mejor futuro, muy asociado a tener mejores condiciones materiales.

2. La culminación de la infancia está determinada por el paso de los niños y las niñas del nivel preescolar al de básica primaria. En esta posición es clara la influencia de la escuela en la concepción de infancia, y justificaría el uso de metodologías y actividades centradas más en el logro de objetivos académicos propuestos, que en las necesidades de desarrollo de los niños y las niñas. Llama la atención que dentro de esta posición se obvia por completo la edad, en cuanto debe haber diferencias en las actividades desarrolladas y las estrategias utilizadas con un niño o una niña de seis años que esté en transición y uno o una de la misma edad que esté en primero de primaria, "porque debe haber otro nivel de exigencia".

En lo concerniente a las diferencias en las concepciones de las educadoras, cabe comentar que los resultados de esta investigación dejan ver la influencia que ejercen los entes educativos rectores en la configuración de diversas concepciones de infancia, de acuerdo con la definición de ciertas características que deben estar presentes en los niños y las niñas. Ésta parece ser la base sobre la que las educadoras otorgan sentido a sus prácticas cotidianas. 


\section{Infancia que se quiere y se forma en valores. Los niños y las niñas}

En las madres comunitarias se evidencia la concepción de niño y niña. Tanto para las educadoras de jardín como para las madres comunitarias, en la concepción de niño es muy fuerte la idea de la importancia de formarlos en valores personales y sociales para que puedan tener un buen futuro y de atenderlos con mucho afecto y dedicación. Contrariamente a lo que se creería, a causa de la escasa formación académica de muchas de ellas y a la creencia bastante difundida en la sociedad de que las madres comunitarias ofrecen una atención que no va más allá del cuidado físico e incluso que allí se pierde el tiempo, la idea de educarlos con afecto tiene gran peso en la promoción del desarrollo de las niñas y los niños, pues según estudios recientes (Friedrich y Preiss, 2003; Rubin, 2000; Acarín, 2003; Ramos, 2002; Katz y Rubin, 2000; Ibarra, 1997, citados por Peralta, 2004) se ha encontrado que:

- El buen aprendizaje (no hecho a la fuerza) aumenta la dopamina y, por ende, produce un sentimiento de felicidad, lo que refuerza a la persona.

- El sistema emocional es el que decide qué estímulos son importantes y valiosos, a través del sistema límbico. Las emociones son energía en movimiento, que estimulan grandes áreas del cerebro, logrando conexiones poderosas. A mayor emoción en el aprendizaje, mayor integración y recuerdo de éste.

- Emoción y motivación son las que dirigen la atención sobre qué informaciones se archivan en los circuitos neuronales.

- Tanto las relaciones humanas significativas, como los gestos afirmativos y la risa, liberan en el cerebro algunos neurotransmisores que producen una sensación de bienestar y favorecen el aprendizaje.

Lo señalado con anterioridad muestra el gran potencial que tiene para los niños y las niñas contar con un ambiente favorable para su desarrollo. Faltaría hacer mayor hincapié en la calidad de las actividades que allí se llevan a cabo para aprovechar realmente dicho ambiente.

\section{Infancia que trabaja académicamente como inversión para un buen futuro. Los alumnos y las alumnas}

En las docentes, la concepción de alumno es más fuerte que la de niña o niño. Las educadoras de transición se orientan también al futuro, pero guiadas por el trabajo académico. En algunas instituciones las exigencias académicas de este nivel se han homologado a las de primero de primaria, con lo que se incrementa la idea de alumno y predomina sobre la de niño y niña, desplazando el interés de las educadoras hacia las exigencias académicas y el cumplimiento de programas. Vale la pena destacar que si la concepción de infancia está más ligada a la idea de un alumno que a la de niño o niña, se tiende a sacrificar el medio amable y afectuoso que tanto valoran las madres comunitarias. 


\section{Infancia que trabaja académicamente y con mucha exigencia como inversión para un buen futuro. Los alumnos y las alumnas deben trabajar duro}

Las educadoras del sector público evidencian concepciones de infancia marcadas por el desarrollo libre y espontáneo que plantea el Ministerio de Educación, pero aclarando que a las niñas y los niños "se les debe exigir mucho" para que puedan tener un mejor futuro. Esta posición sugiere inquietudes en torno a qué tanto el "exigir mucho" tiene implicaciones en la eliminación de las condiciones adecuadas para aprender, expuestas en el primer numeral de este apartado. A esto se suma la idea de la necesidad de exigencia, fundada en la suposición de que por pertenecer a estratos bajos, los niños y las niñas no cuentan con las condiciones personales requeridas para lograr lo que se proponen las educadoras, quienes ven en la educación una posibilidad de movilidad social para sus estudiantes.

\section{Infancia que se adecua a los planteamientos filosóficos de las instituciones. Los alumnos y las alumnas se acomodan a las instituciones}

Las educadoras del sector privado adjudican a los niños y las niñas características relacionadas con la filosofía del colegio en que trabajan. Estas instituciones varían en sus planteamientos y, por supuesto, tienen un interés claro de posicionamiento en el ámbito educativo. Las hay desde posiciones de respeto a los procesos de desarrollo de las niñas y los niños, haciendo hincapié en su bienestar y comodidad, hasta aquellas que basan su oferta en la promesa de anticipar desarrollos y logros de los pequeños. Obviamente, las estrategias y actividades educativas se orientan a ello y las educadoras asumen la posición institucional, adaptando su trabajo a los planteamientos que ésta hace sobre los niños y las niñas.

\section{A modo de conclusión}

Las ideas expuestas anteriormente señalan que si bien las concepciones de infancia tienen su base en el universo simbólico de las culturas, la institución escolar afecta este universo, generando distintas concepciones de infancia.

De esta manera, encontramos soporte para asegurar que es posible transformar las concepciones de infancia a través de la formación teórica y práctica de los educadores de la primera infancia, en el planteamiento de Zimmerman y Gerstenhaber (2000), ya citado antes en este documento, en el que plantean:

Por concepción se entiende un proceso personal por el cual un individuo estructura su saber a medida que integra sus conocimientos. Este saber se elabora, en la mayoría de los casos, durante un periodo bastante amplio de la vida, a partir de su arqueología; es decir, de la acción cultural parental, de la 
práctica social del niño en la escuela, de la influencia de los diversos medios de comunicación y, más tarde, de la actividad profesional y social del adulto ${ }^{7}$. Las concepciones personales son la "única trama de lectura" a las que se puede apelar cuando se confronta con la realidad (p. 125)

Estas concepciones tienen consecuencias directas en las prácticas educativas, pero a su vez las prácticas pueden ser una fuente o bien de consolidación de las concepciones existentes, o bien de modificación de ellas. En esta interdependencia entre concepciones de infancia, configuradas socialmente y por medio de las prácticas educativas, es importante explorar relaciones como las siguientes:

- La escuela es preparación para el futuro-la infancia es preparación para la vida.

- Niveles escolares-niños y alumnos: ¿categorías excluyentes?

Uno de los caminos que abren esta investigación a otras futuras es profundizar en la comprensión de cómo desde la escuela y otras instituciones educativas se generan concepciones de infancia derivadas de sus políticas, programas, proyectos, contextos institucionales y cotidianidad en las aulas de clase y salas del nivel inicial, que van enraizándose en los educadores hasta integrarse a sus cosmovisiones, para llegar a constituirse en nuevos y distintos sistemas de referencia en los cuales se apoyan para tomar decisiones sobre las formas de atender y educar a la infancia.

Así mismo, se convierte en un reto para las entidades encargadas de la formación de los educadores de primera infancia indagar por qué la teoría que se aporta a lo largo de la formación académica llega a tener tan poco peso en las prácticas cotidianas de atención a los niños y las niñas, al igual que en la configuración de concepciones de infancia. ¿Es poco pertinente? ¿Tiene escasa relación con la práctica? ¿Se enfatiza más en los aspectos formales que en la infancia? ¿Se plantean preguntas sobre quién es un niño y quién es una niña? ¿Se nutre la formación teórica y práctica de los educadores de los resultados de investigaciones sobre la infancia? ¿Se obtiene todo el provecho posible de las prácticas? ¿Cómo se evalúan las prácticas? ¿Se toma en cuenta el enorme potencial que tienen las prácticas en la configuración de las concepciones de infancia? La respuesta a estas y otras preguntas es el compromiso de quienes trabajamos en la investigación y en la formación de la infancia, así como de los educadores que la atienden.

\footnotetext{
${ }^{7}$ La cursiva es nuestra.
} 


\section{Bibliografía}

Abric, J. (2001). Prácticas sociales y representaciones. México: Ediciones Coyoacán y Presses Universitaires de France. Colección Filosofía y Cultura Contemporánea.

Bonilla, E. y Rodríguez, P. (2000). Más allá del dilema de los métodos. Bogotá: Norma.

Colombia, Ministerio de EducaCIÓN Nacional, MEN. (1996). Hacia la comprensión del nivel de educación preescolar desde el espíritu de la Ley General de Educación. Bogotá: Ministerio de Educación Nacional.

Delgado, B. (2000). Historia de la infancia. Barcelona: Ariel.

Ferreiro, E. y Teberosky, A. (1979). Los sistemas de escritura en el desarrollo del niño. México: Siglo XXI Editores.

GRUPO DE INVESTIGACIÓN EN InFANCIA. Facultad de Educación. Universidad de La Sabana. (2004-2007). Documentos de trabajo (circulación interna). Bogotá.

Guzmán, R. (2007, 28 a 31 de mayo). Concepciones de infancia que tienen los Educadores del nivel inicial en Chía. Ponencia presentada en el I Seminario Internacional y VI Nacional de Investigación en Educación y Pedagogía. Bogotá: Universidad Pedagógica Nacional.

Instituto Colombiano de Bienestar Familiar, IcbF. (2003). Primera infancia y desarrollo. El desafío de la década. Bogotá: Icbf.

Muñoz, C. y Pachón, X. (1991). La niñez en el siglo XX. Bogotá: Planeta.
Peralta, M. (2003). Los desafíos de la educación infantil en el siglo XXI y sus implicaciones en la formación y prácticas de los agentes educativos. En ICBF, Primera infancia y desarrollo. El desafío de la década. Bogotá: Icbf.

Peralta, M. (2004). Nacidos para aprender. Buenos Aires: Editorial Infanto Juvenil.

Postman, N. (2004). Los incunables de la niñez. En Giros y reveses: representaciones de la infancia a lo largo de la historia. Venezuela: Editorial Arte.

Sáenz, J., Saldarriaga, O. y Ospina, A. (1997). Mirar la infancia: pedagogía, moral y modernidad en Colombia, 1903-1946. Medellín: ColcienciasEdiciones Foro Nacional por Colombia -Uniandes-Editorial Universidad de Antioquia.

Umayahara, M. (2003). Proyecto regional de indicadores de la primera infancia. En ICBF, Primera infancia $y$ desarrollo. El desafío de la década. Bogotá: Icbf.

Zimmerman, M. y GerstenHABer, C. (2000). Acerca del enseñar y del aprender: una aproximación a las concepciones docentes en el nivel inicial. En Nora Elichiry. Aprendizaje de niños y maestros. Buenos Aires: Editorial Manantial.

Zuluaga. O. (1987). Pedagogía e historia. Bogotá: Ediciones Foro Nacional por Colombia. 